\title{
A system for computer control of auditory stimuli
}

\author{
STEVEN E. POLTROCK and NANCY N. MATHEWS \\ University of Washingtom, Seattle, Washington 98195
}

\begin{abstract}
A computer system for generation of auditory stimuli is described. The system produces nat ural-speech or software-generated stimuli for monaural, binaural, or dichotic presentation. Stimuli have been generated for experiments run both on-line and off-line.
\end{abstract}

In the human experimental laboratory, flexible control and precise timing of visual stimuli can be easily accomplished through use of a computer-controlled CRT or slide projector. In contrast, accurate control of auditory stimuli is difficult to achieve, particularly when speech sounds are involved. The present paper describes a computer system, developed at the University of Washington, that permits precise control of a wide variety of auditory stimuli.

The generation of tones, noise, and other mathematically specifiable waveforms is easily accomplished without use of computers. However, most common methods of stimulus generation do not permit accurate control of the timing of natural-speech sounds or dichotic presentation. Stimuli consisting of naturalspeech sounds are usually recorded on tape by a practiced speaker, with the interval between stimulus onsets controlled by observation of a metronome. Production of stimuli for dichotic presentation introduces the further problem of synchronizing the two tape channels. Manual means of achieving synchronization are laborious and inaccurate. Knight and Kantowitz (1973) described a computer system that accurately synchronizes the onsets of dichotic stimuli, but does not permit an onset asynchrony between channels or accurate control of the interval between dichotic pairs.

The present system will accurately reproduce a wide range of stimuli for monaural, binaural, or dichotic presentation. Most audio sources, including microphones, tape decks, and signal generators, may provide inputs to the system. In addition, stimulus waveforms may be created by software functions. The interval between stimulus onsets, or stimulus onset asynchrony (SOA), on the same channel or between channels is specified to the nearest millisecond.

\section{HARDWARE DESCRIPTION}

The system programs are implemented on a Data General Corporation NOVA 800 minicomputer with

Development of this system was supported by the National Institute of Mental Health, Grant MH 21795 (Earl Hunt, principal investigator), and the National Institute of Education, Grant NIE-G-74-0104 (Clifford Lunneborg, principal investigator), to the University of Washington.
$24 \mathrm{~K}$ of central memory. One channel of 10-bit analogto-digital converter (ADC) digitizes input waveforms, while two 8-bit digital-to-analog converters (DACs) output the digitized stimuli. Seven-track magnetic tape serves as mass storage for the digitized stimuli. The programs and command files specifying the order and timing of stimuli are stored on a Ball Computer Corporation disk cartridge. Other equipment used with the system includes a microphone, amplifier, stereo tape recorder, oscilloscope, and an Infoton video terminal.

\section{GENERAL SYSTEM DESCRIPTION}

A large storage capacity, high data rates, and precise timing are required for generation and control of auditory stimulus events. Data rates and storage requirements are dictated by the data conversion sampling rate, which in turn controls the upper bandpass limit of the system. With a $10-\mathrm{kHz}$ sampling rate, the system can reproduce signal components below $5 \mathrm{kHz}$ (adequate for speech), but 10,000 data values are required for each second of input or output. This storage requirement is doubled for two-channel applications. To facilitate meetIng these requirements, the system does not run on-line. Instead, an audio tape is generated that may be used either on-line or off-line. The system has the disadvantage of preventing stimulus manipulations contingent upon a subject's responses, but has the advantages of reducing storage and processing overhead.

The production of a stimulus tape involves two stages. In the first stage, a library of natural-speech or software-generated stimuli is constructed and stored on magnetic tape. The second stage accesses the library entries and records the stimuli on audio tape. The experimenter controls the stimulus order, tape channel, and SOA by specifying these parameters in a command file.

\section{LIBRARY CONSTRUCTION}

The library created by the first stage of processing consists of a single file on magnetic tape. Each record of the file contains the information necessary to reproduce one stimulus. The record includes a stimulus identification number assigned by the experimenter, a count of 
the number of data bytes in the record, and as many as 10,0008 -bit values that represent the stimulus waveform when output at a $10-\mathrm{kHz}$ rate. Separate programs have been developed for constructing libraries of naturalspeech sounds and software-generated tones.

\section{Natural-Speech Sounds}

A tape library of natural-speech sounds, or any sounds input by an audio device, is created by the experimenter through a program, TALK, written in NOVA assembly language. The hardware configuration required by TALK is schematized in Figure 1. TALK provides the experimenter with commands, entered via the video terminal, for reading, writing, or positioning the library tape, creating a new stimulus, assigning an identification number to the stimulus, and adjusting the position of a speech sound within the sampling interval. The command format is a single letter representing the command, or a letter and numeric parameter. A stimulus library is created by repetitively inputting a stimulus via the ADC, adjusting the position of the stimulus within the sampling interval, and writing the stimulus and stimulus number on the library tape. An end-of-file mark completes the library file.

The stimuli may be input directly to the $\mathrm{ADC}$ via a microphone and amplifier. However, it is usually preferable to prerecord a list of the stimuli in an environment that permits careful monitoring of stimulus intensity and background noise. When the stimuli are input to the system, the input voltage level is monitored with an oscilloscope to insure that the ADC $\pm 1-V$ limit is not exceeded.

Inputting a stimulus. The stimulus input command is always accompanied by a stimulus number. TALK responds to the command by prompting the experimenter with the message, "Strike key when ready." A video terminal keypress signals TALK to start the sampling interval. The input to the ADC is continuously monitored until a value exceeds a threshold of $\pm 20 \mathrm{mV}$. When the stimulus onset is sensed, the input to the ADC is sampled for $1 \mathrm{sec}$ at a rate of $10 \mathrm{kHz}$. The lowest order 2

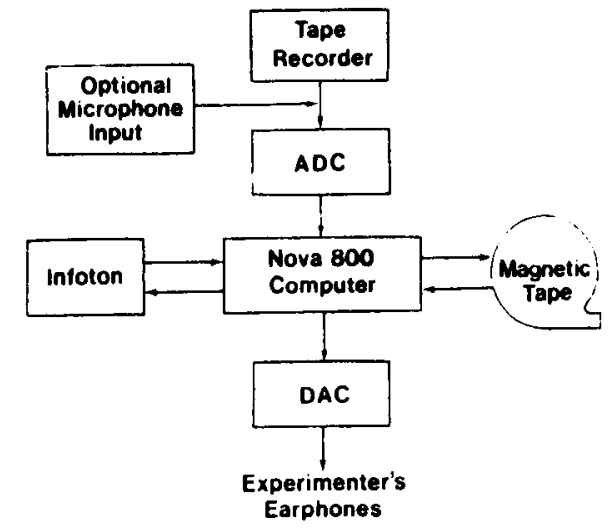

Figure 1. Block diagram of hardware configuration for program TALK. bits of the 10-bit samples are deleted to match the resolution of the 8-bit DACs. TALK packs two successive 8-bit samples in each 16-bit computer word, reducing the core requirements for a $1-\mathrm{sec}$ sample to 5,000 storage locations.

The $10-\mathrm{kHz}$ sampling rate is accomplished by instruction counting. That is, the instruction to sample the ADC is imbedded in a program loop consisting of enough instructions to require 100 microsec of processing time.

Adjustment of stimulus position. Since stimuli are often shorter than the 1 -sec sampling interval, commands are available to adjust the position of the stimulus within the sampling interval by eliminating sampie points at the beginning or end of the interval. If background noise prevents use of the $20-\mathrm{mV}$ threshold for detection of the stimulus onset, the experimenter may search for the stimulus onset in the stored $1-\mathrm{sec}$ sample by inputting larger threshold values. All stimulus samples following the first sample that exceeds the threshold are output through a DAC. The experimenter determines whether the input threshold discriminated between signal and noise by listening over earphones. If noise precedes the stimulus, then a larger threshold is input. If the stimulus was truncated, then the threshold is decreased. Typically, a series of threshold values is input until the smallest threshold value is found that eliminates all samples prior to the speech sound. When a threshold has been selected, a command is entered to delete all samples prior to the detected onset.

If the speech sound is considerably shorter than the remaining sampling interval, the experimenter may wish to eliminate samples from the end of the interval. A command permits the experimenter to input a maximum duration in milliseconds.

Library tape input/output. A single command causes TALK to write the stimulus number, a byte count, and the digitized speech stimulus as a single record on the library tape. With other commands, the user can forward space or back space the tape, read a library stimulus from the tape, or write an end-of-file mark.

\section{Software-Generated Tones}

A program, TONE, written in FORTRAN produces a stimulus library of software-generated sine functions. The experimenter supplies the stimulus identification number, frequency, duration, and rise and fall time for each tone. The tones are mathematically computed and output to the library tape in the required format. The TONE program is easily extended to generate other mathematical functions such as triangular waves, square waves, or white noise.

\section{RECORDING AN AUDIO TAPE}

A program, TAPE, written in assembly language 


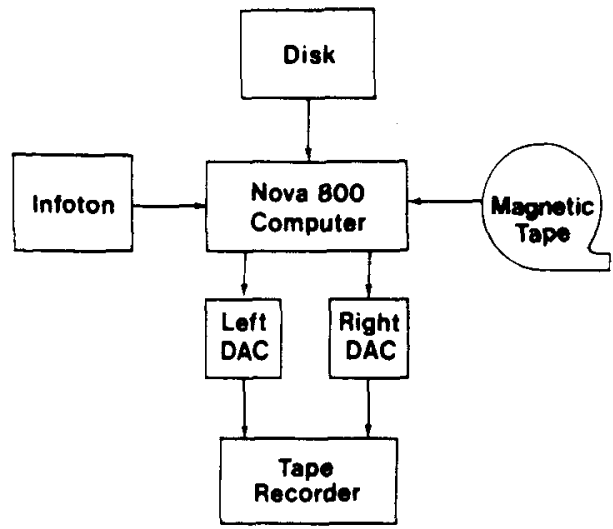

Figure 2. Block diagram of hardware configuration for program TAPE.

controls the recording of the stimuli on an audio tape. The hardware configuration required by TAPE is schematized in Figure 2. The stimulus order, tape channel, and timing are controlled by the experimenter through a disk command file. The command file consists of a list of the stimulus identification numbers, the tape channel for each stimulus, and the SOAs in milliseconds. Simultaneous presentation of dichotic stimulus pairs is accomplished by specifying an onset interval of $0 \mathrm{msec}$ between stimuli on opposite channels. The command file may be created through use of an Editor or written by a FORTRAN program.

The TAPE program reads the stimuli from the library in the order specified by the command file and outputs each stimulus to a DAC. The timing required to maintain the stimulus output rate and to adjust the interval between stimuli is accomplished by instruction counting. In parallel with the stimulus output loop, library stimuli are input in the order specified by the command file. The routines controlling input from the library are activated when an interrupt is received from the tape drive or when output from a stimulus buffer is completed. The input routines maintain four stimulus buffers, providing double buffers for dichotic stimuli or quadruple buffering for monaural stimuli.

In spite of the use of multiple buffering, the time required to access library stimuli imposes a lower limit on the interstimulus interval in a stimulus list. Production of the stimuli off-line permits use of a method that effectively halves this lower limit. Stimulus values are output at $5,000 \mathrm{~Hz}$, half the sampling rate used by TALK, and recorded at a tape speed of 3.75 ips. When the tape is played back at $7.5 \mathrm{ips}$, the original stimulus rate and signal frequencies are recovered.

\section{TIMING ACCURACY}

Instruction counting provides a very accurate means of timing events less than $1 \mathrm{msec}$ in duration. However, the technique suffers from the disadvantage of sensitivity to cycle stealing. Cycle stealing refers to the use of instruction cycles by a peripheral performing a memory access. If the library tape is read while a stimulus is being output, cycle stealing results in a $3 \%$ decrease in the stimulus frequency. In order to correct this error, each time the tape is read the TAPE program adjusts the counters used to control the timing.

To determine the timing accuracy of TALK and TAPE, stimuli consisting of $1 \mathrm{kHz}$ sine waves were generated both by TONE and by sampling the output of a signal generator with TALK. The functions output at the DACs were displayed on a Tektronix dual trace storage oscilloscope, permitting detection of errors greater than or equal to $1 \%$. No error in the frequency of either function was detectable. When stimuli were simultaneously output to both DACs, again no error in frequency was detectable. When an interval between stimulus onsets of 1,2 , or $10 \mathrm{msec}$ was imposed, no error in the interval was detectable.

\section{APPLICATIONS}

Both on-line and off-line applications for this system have developed in the few months of its existence. Off-line applications include generation of lists for a stimulus-suffix experiment with varied interstimulus intervals, a selective-attention task requiring dichotic presentation of 19-word lists, and tasks designed to measure degree of phonetic fusion and temporal order discriminability during dichotic listening. On-line applications require that one channel of the auditory tape contain timing marks generated by the system, such as a square wave. A simple signal detector closes a computer-monitored contact in response to the timing mark providing synchronization with visual displays. In an auditory recognition-masking experiment, the computer records responses and provides visual feedback. Cross-modality temporal order judgement tasks are also planned.

\section{REFERENCE}

KNight, J, L., JR., \& Kantowitz, B. H. A minicomputer method for generating dichotic word pairs. Behavior Research Methods \& Instrumentation. 1973, 5. 231-234. 